\title{
Thank You for Your Service!
}

Lindsey E. Eberman, PhD, LAT, ATC

Indiana State University, Terre Haute, IN

\section{Thank You for Your Service!}

The editorial board wants to thank all those who serve or have served Clinical Practice in Athletic Training, this year. As we continue to evolve, we have had several Section Editors move on, while we are honored to welcome new Section Editors. Let us first thank Brian Vesci, Esther Nolton, and Alison O'Connor Sutherland for their service to the Journal. Thank you for your continued support, contributions, and dedication to clinical practice research. And, a great welcome to Tim Nicollelo (Disablement Model Case Studies and Reports), S. Andrew Cage (Validation Case Studies and Reports), Kim Barber Foss (Point-of-Care Research), and Nick Pfeifer (Quality Improvement Reports).

We continue to evolve as a Journal and we are happy to announce Dr. Zachary Winkelmann will be joining the Senior Editorial Board and Dr. Matthew Drescher and Ms. Kelcey Granger will be joining the team as Staff Editors. We are hopeful that these small changes will help better support Section Editors, Reviewers, and Authors.

Below we recognize our section editors and reviewers who have contributed to the Journal over the past year:

\section{SECTION EDITORS:}

Disablement Model Case Studies and Reports

Tim Nicollelo, MS, LAT, ATC -St. Luke's Health System

\section{Validation Case Studies and Reports}

S. Andrew Cage, MEd, LAT, ATC - University of Texas Tyler

\section{Point-of-Care Research}

Kim Barber Foss, MA, LAT, ATC -Cincinnati Children's Hospital

Clinical Outcomes Research

JJ Wetherington, MS, LAT, ATC - St. Luke's Sports Medicine

Quality Improvement Reports

Nick Pfeifer, EdM, ATC - Boston University

Preceptor Case Studies

Zachary Dougal, DAT, LAT, ATC - Ball State University

Evidence-to-Practice Reviews

Cameron Powden, PhD, LAT, ATC - Indiana State University

\section{Clinical Expertise Commentary}

Hollie Walusz, MA, ATC, PES - Boston University

Patient-Centered-Care Commentary

Mark Laursen, MS, ATC, Boston University

\section{REVIEWERS:}

Nicholas C Merritt, DAT, ATC, SCAT - Furman University

Skye Livermore-Brasher MPA, LAT, ATC Columbus State University

Samantha Jane Atkielski MS, LAT, ATC, CES University of Wisconsin Milwaukee 
Kevin Michael Schroeder, DAT, ATC - University of New Mexico

Connor Burton, DAT, LAT, ATC - Indiana State University

Zachary K Winkelmann, PhD, SCAT, ATC University of South Carolina

Justin Tatman, MS, LAT, ATC - TRIA Orthopedic Center

Danielle Colegrove - Northwestern University

Vicky Graham, DAT, ATC - Xavier University

Jamie Nikander, DAT, LAT, ATC - West Olympia Sports Medicine

Emily Gibb, MA, ATC - Boston University

Lucas Bianco, MS, LAT, ATC - University of Lynchburg

Sara J. Brown, MS, ATC - Boston University

Daniel Walen, DAT, AT, ATC - Western Michigan

University

Thomas Abdenour, DHSc, ATC, CES - Retired

Adam Babiarz, DAT, LAT, ATC - ATI Physical Therapy

Nicholas DePhillipo, MS, ATC, OTC, CSCS - The Steadman Clinic

Kelcey Granger MS, LAT, ATC - Indiana State University

Chyrsten Gessel MS, LAT, ATC - Mariette College

Savannah Katelyn Bailey, LAT, ATC - United States Marine Corps

Alli Zeigel Powell, DAT, AT - Colorado Mesa University

Steve Middleton, DPT, ATC - William Woods University
McCall Christian, DAT, LAT, ATC - Missouri State University

Richelle Williams, PhD, ATC - Drake University

Kim Mace, DAT, ATC - Boston University

Katie Knappenberger, MS, RD, CSSD, ATC Northwestern University

Nancy Uriegas, MS, SCAT, ATC - University of South Carolina 